Article

\title{
Quasi-3D Plasmonic Nanowell Array for Molecular Enrichment and SERS-Based Detection
}

\author{
Sunho Kim ${ }^{1}$, Chaewon Mun ${ }^{2}$, Dae-Geun Choi ${ }^{3}$, Ho Sang Jung ${ }^{2}$, Dong-Ho Kim ${ }^{2, *}$, \\ Shin-Hyun Kim ${ }^{1, *}$ and Sung-Gyu Park ${ }^{2, *}$ (D) \\ 1 Department of Chemical and Biomolecular Engineering, Korea Advanced Institute of Science and \\ Technology, Daejeon 34141, Korea; shkim1020@kaist.ac.kr \\ 2 Advanced Nano-Surface Department (ANSD), Korea Institute of Materials Science (KIMS), Changwon, \\ Gyeongnam 51508, Korea; apple1025@kims.re.kr (C.M.); jhs0626@kims.re.kr (H.S.J.) \\ 3 Nano-Mechanical Systems Research Division, Korea Institute of Machinery \& Materials (KIMM), \\ Daejeon 305-343, Korea; lamcdg@kimm.re.kr \\ * Correspondence: dhkim2@kims.re.kr (D.-H.K.); kim.sh@kaist.ac.kr (S.-H.K.); sgpark@kims.re.kr (S.-G.P.); \\ Tel.: +82-55-280-3632 (S.-G.P.)
}

Received: 8 April 2020; Accepted: 29 April 2020; Published: 14 May 2020

\begin{abstract}
We report on a quasi-three-dimensional (3D) plasmonic nanowell array with high structural uniformity for molecular detection. The quasi-3D plasmonic nanowell array was composed of periodic hexagonal Au nanowells whose surface is densely covered with gold nanoparticles (Au NPs), separated by an ultrathin dielectric interlayer. The uniform array of the Au nanowells was fabricated by nanoimprint lithography and deposition of Au thin film. A self-assembled monolayer (SAM) of perfluorodecanethiol (PFDT) was coated on the Au surface, on which Au was further deposited. Interestingly, the PFDT-coated Au nanowells were fully covered with Au NPs with an ultra-high density of $375 \mu \mathrm{m}^{-2}$ rather than a smooth film due to the anti-wetting property of the low-energy surface. The plasmonic nanogaps formed among the high-density Au NPs led to a strong near-field enhancement via coupled localized surface plasmon resonance and produced a uniform surface-enhanced Raman spectroscopy (SERS) response with a small relative standard deviation of 5.3\%. Importantly, the highly uniform nanostructure, featured by the nanoimprint lithography and 3D growth of densely-packed Au NPs, minimizes the spatial variation of Raman intensity, potentially providing quantitative analysis. Moreover, analyte molecules were highly concentrated and selectively deposited in nanowells when a water droplet containing the analyte was evaporated on the plasmonic substrate. The analyte formed a relatively thick overcoat in the nanowells near the triple line due to the coffee-ring effects. Combining 3D plasmonic nanowell substrates with molecular enrichments, highly sensitive detection of lactic acid was demonstrated. Given its combination of high sensitivity and signal uniformity, the quasi-3D plasmonic nanowell substrate is expected to provide a superior molecular detection platform for biosensing applications.
\end{abstract}

Keywords: nanoimprint lithography; 3D plasmonic nanowell array; surface-enhanced Raman spectroscopy; molecular concentration; signal uniformity

\section{Introduction}

Plasmonic nanostructures provide strong light localization, through which the electromagnetic field is drastically enhanced in the nanogaps known as "hotspots". This phenomenon has led to the development of an ultrasensitive molecular sensing method referred to as surface-enhanced Raman spectroscopy (SERS) [1-9]. The SERS detection technique has been used in medical diagnosis [10,11], environmental monitoring, and food safety control because it enables the sensitive, rapid, and 
label-free identification of molecules. The SERS intensity is highly sensitive to the arrangement, size, and topography of the plasmonic nanostructures. Therefore, the production of precisely controlled uniform metal nanostructures is very important to guarantee reliable molecular detection and quantitative analysis. Top-down approaches usually provide uniform and reproducible nanostructures in comparison with bottom-up approaches. The conventional top-down nanofabrication techniques to produce accurate structural arrangement and geometries for SERS applications include electron-beam lithography [12,13] and laser-interference lithography [14-20]. Although these techniques enable the elaborate construction of plasmonic nanostructures, the low-throughput and high-cost of production restrict practical uses. Furthermore, it is difficult to produce 3D nanostructures with the technique albeit the 3D geometries are highly desired to enhance sensitivity. Therefore, a robust and scalable fabrication method that simultaneously achieves high production-throughput, low cost, and high sensitivity of Raman measurement remains very important for commercializing prominent plasmonic technologies.

Nanoimprint lithography (NIL) is a simple, reproducible, and scalable method to create plasmonic nanostructures over a large area so that it has been used for fabrication of SERS substrates [21-25]. The NIL enables the production of various periodic nanostructures on both rigid and flexible substrates. However, it is difficult to produce plasmonic nanostructures with densely packed sub- $10 \mathrm{~nm}$ nanogaps for ultrasensitive sensing through the NIL itself. For example, Chou et al. [26] modified nanocompact disks with $10 \mathrm{~nm}$ features by NIL. To further enhance Raman intensity, on the other hand, it has been suggested to concentrate molecules in the nanogaps. Therefore, it is highly demanded, for the development of pragmatic sensing systems, to fabricate plasmonic nanostructures with a high density of nanogaps, through the NIL, that can concentrate and position probe molecules at the hotspots.

Here, we report on a 3D plasmonic hexagonal nanowell array with densely-packed $\mathrm{Au}$ nanoparticles (NPs) on the entire surface of Au nanowells, separated by a nanoscale-thick dielectric layer. The uniform hexagonal nanowell array with a well diameter of $400 \mathrm{~nm}$ and pitch of $500 \mathrm{~nm}$ was fabricated by NIL and a thin film of Au is deposited on the surface. The Au surface of the nanowell array was further decorated by a high density of Au NPs through the 3D growth of Au islands on $1 \mathrm{H}, 1 \mathrm{H}, 2 \mathrm{H}, 2 \mathrm{H}$-perfluorodecanethiol (PFDT)-coated, low-energy surface. The deposition condition of $\mathrm{Au}$ NPs was optimized through a finite-difference time-domain (FDTD) simulation and experiments to maximize the electric field enhancement and Raman signal intensity. The signal uniformity of the optimized plasmonic nanostructure was thoroughly evaluated through a microRaman mapping technique, which revealed that the signal intensity had a very small relative standard deviation of $5.3 \%$. The nanowells can accommodate water and accumulate analyte molecules on their surface during the evaporation of a water droplet. In particular, analyte was highly concentrated to form a relatively thick overcoat on the Au NPs in nanowells near the triple line of the droplet. Combining 3D plasmonic nanowell substrates with molecular enrichments, highly sensitive detection of lactic acid, an excellent indicator of muscle tolerance, was demonstrated. The limit of detection (LOD) was measured to be $0.05 \mathrm{mM}$, which was 6 times lower than the LOD in the previous study [27].

\section{Materials and Methods}

\subsection{Fabrication of Gold Nanoparticle (Au-NP)-Decorated Nanowell Arrays}

A nanowell array in polymer substrates was doubly replicated from a Si master pattern with a hexagonal nanowell array through two steps of the imprinting process. Before the imprinting, the $\mathrm{Si}$ master mold was coated with an anti-adhesion molecule of hexamethyldisilazane (Sigma-Aldrich, Seoul, Korea) through vapor deposition to facilitate demolding. For both the first and second imprinting, an ultraviolet (UV)-curing resin (MINS-311RM, MINUTA Tech, Osan, Korea) were spin-coated onto a polyethylene terephthalate (PET) film for $30 \mathrm{~s}$ at a spin speed of $1000 \mathrm{rpm}$ and the mold was pressed against the PET sheet under a pressure of 100 bar for $600 \mathrm{~s}$ using nanoimprinting equipment for a 5-inch wafer scale. After the double replication, a $100 \mathrm{~nm}$-thick Au film was then directly deposited at the rate of $2.0 \AA /$ s onto the polymer nanowell pattern using a sputtering system (SNTEK, Co. Ltd., 
Suwon, Korea). The Au nanowell substrate was then incubated for $2 \mathrm{~h}$ in a square dish to form a PFDT self-assembled monolayer (SAM). Afterward, another Au layer was deposited onto the PFDT-coated Au nanowell array at the rate of $0.3 \AA$ /s using thermal evaporation (SNTEK, Co. Ltd., Suwon, Korea), which resulted in quasi-3D plasmonic nanowell substrates.

\subsection{Characterization and Surface-Enhanced Raman Spectroscopy (SERS) Measurements}

Surface morphologies were characterized by field-emission scanning electron microscopy (FE-SEM; JSM-6700F, Jeol, Tokyo, Japan). The SERS spectra of methylene blue (MB) were recorded using a handheld Raman spectrometer (CBEx, Snowy Range Instrument, Laramie, WY, USA) with a laser wavelength of $633 \mathrm{~nm}$ and $785 \mathrm{~nm}$, and laser power of $10 \mathrm{~mW}$. A $3 \mu \mathrm{L}$ of $5 \times 10^{-6} \mathrm{M}$ MB solution was dropped on the surface of the 3D SERS substrate and the solvent was evaporated. For microRaman mapping, the 3D plasmonic nanowell substrate was dipped into $1 \mathrm{mM}$ benzenethiol (BT) solution for $24 \mathrm{~h}$ for complete surface coverage of probe molecules and the substrate was then washed with ethanol several times and dried gently under $\mathrm{N}_{2}$ gas. The microRaman mapping spectra were obtained using a high-resolution dispersive Raman microscope (Horiba Jobin Yvon, LabRAM HR, Kyoto, Japan) with $50 \times$ objective lens $(\mathrm{NA}=0.75$ ) and a $632.8 \mathrm{~nm}$ HeNe laser. A $16 \mu \mathrm{m} \times 16 \mu \mathrm{m}$ region was measured at $1 \mu \mathrm{m}$ resolution with a laser power of $0.4 \mathrm{~mW}$.

\subsection{Numerical Simulations}

Electromagnetic simulations of quasi-3D plasmonic nanowell structures were performed with the commercial finite-difference time-domain (FDTD) software package (version 8.21, Lumerical Solutions, Seoul, Korea). The nanostructure was sketched based on scanning electron microscope (SEM) observations using Autodesk Fusion 360, and the data were subsequently exported into FDTD software. A linearly polarized plane wave with a wavelength of $633 \mathrm{~nm}$ was incident to the Au nanowell. Because the Au film on the UV-resin surface was $100 \mathrm{~nm}$ thick, we assumed that no light was transmitted through it. The dielectric constant of Au was set to $\varepsilon_{\mathrm{Au}}=-8.962364+1.164 i$ at $633 \mathrm{~nm}$, and the refractive index of PFDT was set to 1.333 [2].

\section{Results and Discussion}

A schematic for the fabrication process of the nanowell array is shown in Figure 1a. A Si master pattern with a hexagonal nanowell array was transferred to a resin-coated PET film; the array has a well diameter of $400 \mathrm{~nm}$, a height of $200 \mathrm{~nm}$, and a pitch of $500 \mathrm{~nm}$. The replicated structure on the PET film had a nanopillar array with the same dimensions as the master pattern (Figure 1b,c). The nanopillar array was replicated again to make a nanowell array with the same structure as the original master mold. The resulting nanohole array is highly uniform (Figure $1 \mathrm{~d}, \mathrm{e}$ ). The polymer nanowell pattern was used for making plasmonic nanowell array with densely-packed Au NPs. The quasi-3D nanowell structures prepared using NIL have several advantages for SERS-based sensing applications: (1) the NIL process is cost-effective and the nanostructures are reproducible; (2) the resulting plasmonic nanostructures exhibit high signal uniformity; and (3) molecules can be accumulated in the plasmonic nanowells to further enhance Raman intensity.

The quasi-3D periodic nanowell structures were prepared by following three steps with the NIL-featured nanowell array. First, a 100 nm-thick Au layer was deposited onto the polymer nanowell array. Second, PFDT SAM was deposited on the Au surface through a vapor-phase deposition. The thiol (-SH) groups of PFDT compactly formed a monolayer at atmospheric pressure because of their strong affinity with the Au layer. The fluoro $(-F)$ groups render the PFDT-coated Au surface to have very low surface energy [5,28]. Third, another Au layer was deposited onto the PFDT-coated Au nanowell array. 
(a)

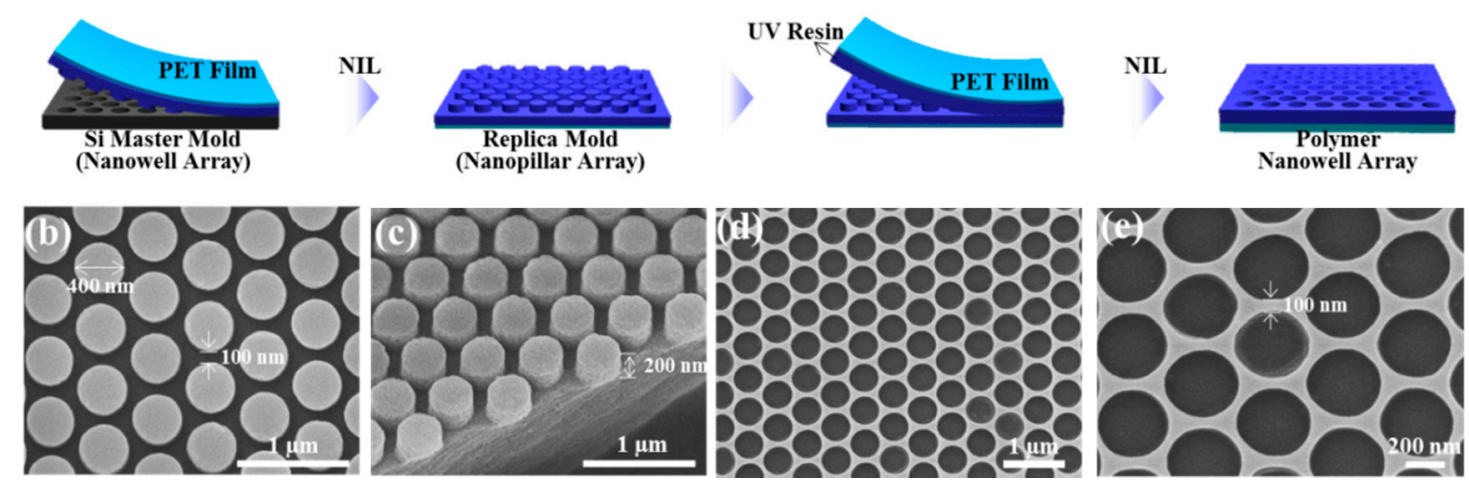

Figure 1. Fabrication of nanowell array. (a) Schematic of the fabrication process of the polymer hexagonal nanowell array using nanoimprint lithography (NIL). (b,c) Scanning electron microscopy (SEM) images showing the top view and $45^{\circ}$-tilted view of a replica mold with a nanopillar array. (d,e) SEM images of polymer nanowell array replicated from the nanopillar soft mold.

Figure 2a shows the nanowell array after the deposition of $\mathrm{Au}$ onto PFDT-coated Au nanowells, where the condition for the formation of a 5-nm-thick Au layer on normal surfaces by conformal deposition was used. Each nanowell contains Au NPs that are distributed on the entire surfaces. The high surface energy of $\mathrm{Au}\left(\gamma_{\mathrm{Au}}=1.54 \mathrm{~J} \mathrm{~m}^{-2}\right)$ and the low surface energy of PFDT $\left(\gamma_{\mathrm{PFDT}}=0.015 \mathrm{~J} \mathrm{~m}^{-2}\right)$ [29] may cause negative spreading parameter, which results in the formation of spherical Au NPs rather than a smooth film on the surface of the PFDT-coated Au. This anti-wetting property of the PFDT SAM caused the formation of Au NPs at 3D growth mode [30] and enabled the formation of dense nanogaps among Au NPs by increasing the size of Au NPs. As the dose of Au deposition increases, so does the size of the discrete Au NPs, resulting in a smaller gap distance (i.e., nanogap) among the Au NPs (Figure 2b). When the condition for the 50-nm-thick Au layer on normal surfaces is used, a dense array of sub-50 nm Au NPs was formed on the entire quasi-3D surfaces of the bottom surface and sidewall of the nanowells and interstitial area among the nanowells (Figure 2c,d). All nanowells showed a high structural uniformity.

We investigated the near-field coupling of the quasi-3D plasmonic nanowell structures using FDTD simulations. For two different deposition conditions of $5 \mathrm{~nm}$-thick and $50 \mathrm{~nm}$-thick Au layers on normal surfaces, the size and spacing of Au NPs were investigated by SEM observation, which were used for the construction of model structures for FDTD simulations; the Au NPs formed on the bottom surface is relatively larger than that on the sidewall of nanowells due to the directional flux of Au atoms during thermal evaporation: Average diameters of Au NPs on the sidewall and bottom surface were $10 \mathrm{~nm}$ and $14 \mathrm{~nm}$ for the condition of a $5 \mathrm{~nm}$-thick Au layer and $30 \mathrm{~nm}$ and $40 \mathrm{~nm}$ for the condition of $50 \mathrm{~nm}$-thick Au layer, respectively. For plane-wave radiation with a wavelength of $633 \mathrm{~nm}$, the electric field was strongly localized in the gaps between the Au NPs and Au film (Figure 2e,f). Interestingly, the gaps on the sidewall show the high near-field enhancement because the gap is aligned parallel to the polarization direction of the incident light. The electric field is one-order of magnitude larger for the condition of the $50 \mathrm{~nm}$-thick Au layer than the condition of the $5 \mathrm{~nm}$-thick Au layer.

Next, we optimized the condition of Au deposition to maximize SERS performance. For this purpose, we used an organic dye, methylene blue (MB), as a probe molecule. A $3 \mu \mathrm{L}$ of $5 \times 10^{-6} \mathrm{M} \mathrm{MB}$ aqueous solution was dropped on the surface of the quasi-3D SERS substrate, and the solvent was evaporated. The SERS signal was then measured using an excitation wavelength of $633 \mathrm{~nm}$ and $785 \mathrm{~nm}$ handheld Raman spectrometers. The quasi-3D nanowell substrates prepared using $50 \mathrm{~nm}$-thick $\mathrm{Au}$ film conditions showed the highly enhanced SERS signal for both laser wavelengths (Figure $2 \mathrm{~g}-\mathrm{j}$ ). This was due to the fact that the quasi-3D nanowell substrates with $50 \mathrm{~nm}$ thick Au film conditions had the highest nanogap density of $375 \mu \mathrm{m}^{-2}$, but the nanogap density decreased for the conditions 
of 75 nm-thick and 100 nm-thick Au films due to fusion of Au NPs, as shown in Figure S1. When the condition for $25 \mathrm{~nm}-, 50 \mathrm{~nm}-$, and $75 \mathrm{~nm}$-thick Au layer on normal surfaces was used, the average diameters of Au NPs were $33 \mathrm{~nm}, 43 \mathrm{~nm}$, and $54 \mathrm{~nm}$, respectively.
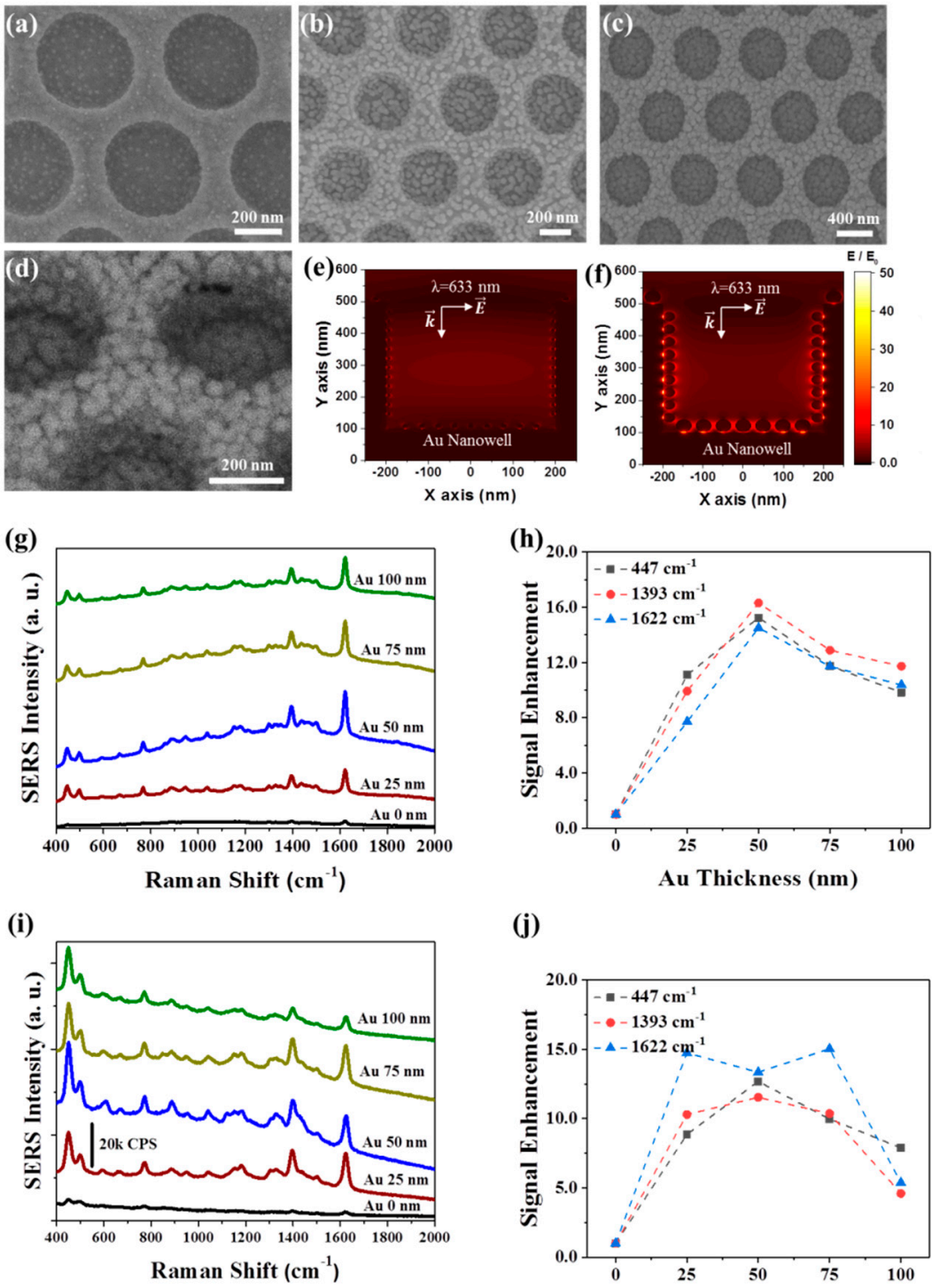

(j)

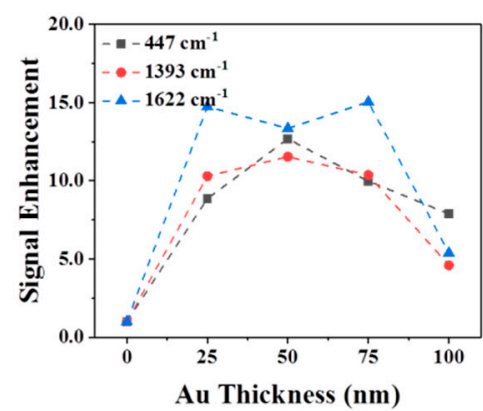

Figure 2. Optimization of Au deposition condition. (a-d) SEM images of the quasi-3D Au nanowell structures with four different $\mathrm{Au}$ deposition conditions, where the conditions for the formation of $\mathrm{Au}$ films with thicknesses of (a) $5 \mathrm{~nm}$, (b) $25 \mathrm{~nm}$, and (c,d) $50 \mathrm{~nm}$ on normal surfaces are used. (e,f) Spatial distributions of electric field intensity in quasi-3D Au nanowells for the conditions of (e) 5 nm-thick and (f) $50 \mathrm{~nm}$-thick Au film formation. The diameters of Au NPs on the sidewall and bottom surfaces and a gap spacing are set to $10 \mathrm{~nm}, 14 \mathrm{~nm}$, and $24 \mathrm{~nm}$ for the condition of $5 \mathrm{~nm}$-thick Au film and those are set to $30 \mathrm{~nm}, 40 \mathrm{~nm}$, and $9 \mathrm{~nm}$ for the condition of $50 \mathrm{~nm}$-thick Au film, based on SEM observation. Raman spectra of methylene blue (MB) taken using the quasi-3D Au nanowells with 5 different deposition conditions of Au deposition using (g) $633 \mathrm{~nm}$ and (i) $785 \mathrm{~nm}$ lasers. Variation of surface-enhanced Raman spectroscopy (SERS) intensity at Raman shifts of $447 \mathrm{~cm}^{-1}, 1393 \mathrm{~cm}^{-1}$, and $1622 \mathrm{~cm}^{-1}$ according to the Au deposition condition for (h) $633 \mathrm{~nm}$ and (j) $785 \mathrm{~nm}$ lasers.

Signal uniformity and reproducibility were very important to guarantee the quantitative analysis. As the quasi-3D nanowells had minimal structural variation nanowell-by-nanowell as confirmed in Figure 2, it was expected that they would show high signal uniformity and reproducibility. To 
investigate this, the substrate was subjected to Raman micromapping (Figure 3). To avoid any variation of molecular concentration and study the influence of structural effect alone, the quasi-3D nanowell substrate was dipped into a $1 \mathrm{mM}$ benzenethiol (BT) solution for $24 \mathrm{~h}$ rather than dropping and drying the solution on the substrate; the dipping made a complete monolayer coating of BT on Au surface. The area of $16 \mu \mathrm{m}(x$-axis) $\times 16 \mu \mathrm{m}$ (y-axis) on the BT-coated substrate was scanned by a resolution of $1 \mu \mathrm{m} \times 1 \mu \mathrm{m}$. Consequently, a total of 256 pixels $(1$ pixel $=1 \mu \mathrm{m} \times 1 \mu \mathrm{m})$ were obtained and the characteristic peaks of BT at 997,1072 , and $1571 \mathrm{~cm}^{-1}$ were used to assess the signal uniformity. Highly uniform distribution of Raman intensities was observed for the quasi-3D nanowell substrate for all three peak positions (Figure $3 \mathrm{a}-\mathrm{c}$ ). According to our statistical analysis, the relative standard deviation (RSD) for the three different peaks was as small as $6.3 \%$ for $997 \mathrm{~cm}^{-1}$ (Figure 3d), 5.6\% for $1072 \mathrm{~cm}^{-1}$ (Figure 3e), and 5.3\% for $1571 \mathrm{~cm}^{-1}$ (Figure 3f). This high signal uniformity is contributed from the minimal structural variation on the substrate as the quasi-3D nanowells were featured by highly-reproducible NIL and growth of high density Au NPs on the low-energy surface.

(a)

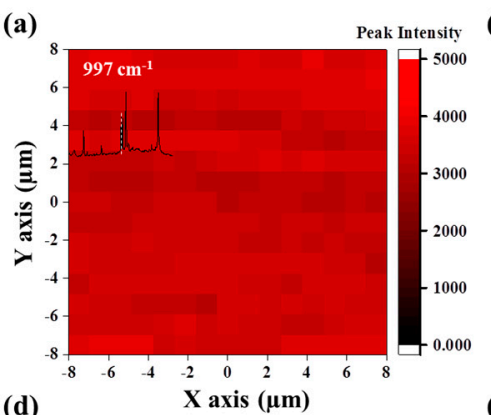

(d)

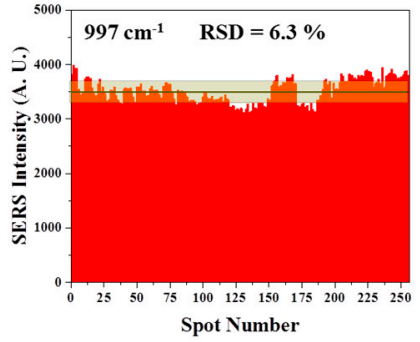

(b)

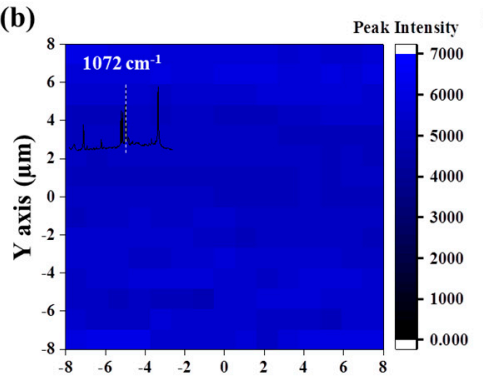

(e)

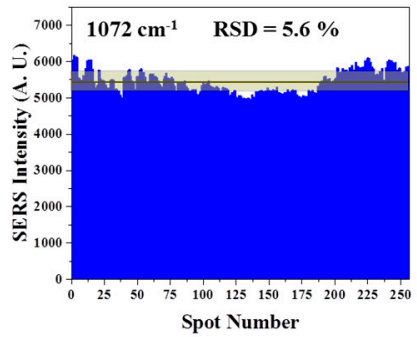

(c)

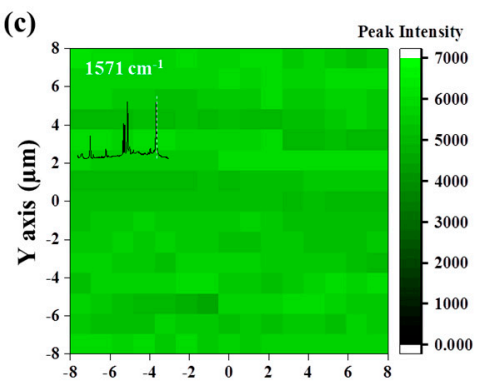

(f)

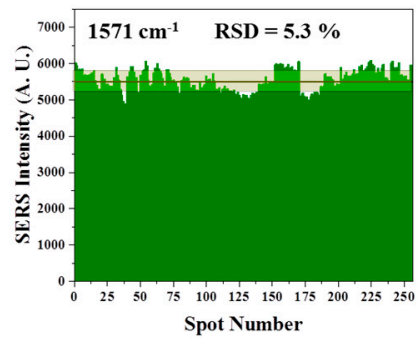

Figure 3. High uniformity of Raman intensity. (a-c) Spatial distribution of Raman intensities of benzene thiol (BT) at characteristic peak positions of (a) $997 \mathrm{~cm}^{-1}$, (b) $1072 \mathrm{~cm}^{-1}$, and (c) $1571 \mathrm{~cm}^{-1}$ acquired by micromapping. (d-f) Variation of Raman intensities on 256 spots. The relative standard deviation (RSD) of the intensity is denoted in each panel.

Because the quasi-3D nanowell structures contain a fluorine-based PFDT interlayer, the surface was hydrophobic. We characterized the hydrophobic properties by dropping an aqueous solution onto the structures, as shown in Figure 4a; the periodic nanowell array developed the vivid green color through wavelength-selective diffraction [31]. The water droplet showed a contact angle (CA) of $134^{\circ}$ (the inset of Figure 4a). When the droplet contained MB, the evaporation of water concentrated MB and left behind a ring-shaped stain due to coffee-ring effect [32]. All nanowells on the central area and rings contained $\mathrm{MB}$ on their surface. In the central area, $\mathrm{MB}$ was deposited on the inner surfaces of the nanowells rather than the interstitial surface among the nanowells (Figure $4 b, c)$; the darker parts in the SEM images have the overcoat of dielectric MB molecules on the Au NPs, whereas bright parts are the Au NPs without the overcoat. This selective deposition in the nanowell is attributed to the trapping of water in the nanowell at the last moment of evaporation due to the transition of wetting state from Cassie-Baxter to Wenzel [2]. The amount of MB deposited in nanowells in the ring-shaped stain was larger than those in the central area, as expected from the coffee-ring effect (Figure 4d-f). Many nanowells appear much darker than those in the central area, which was caused by the formation of a relatively thick MB overcoat in the nanowells (denoted with white circles in 
Figure 4e). The molecular enrichment and selective deposition in nanowells potentially provide highly sensitive molecular detection for water-soluble molecules.
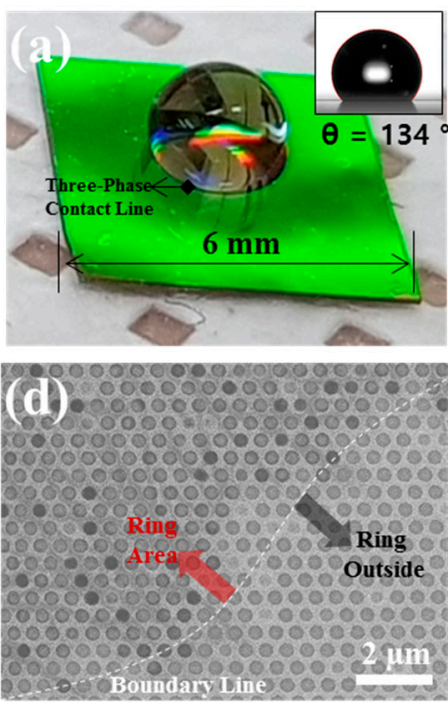
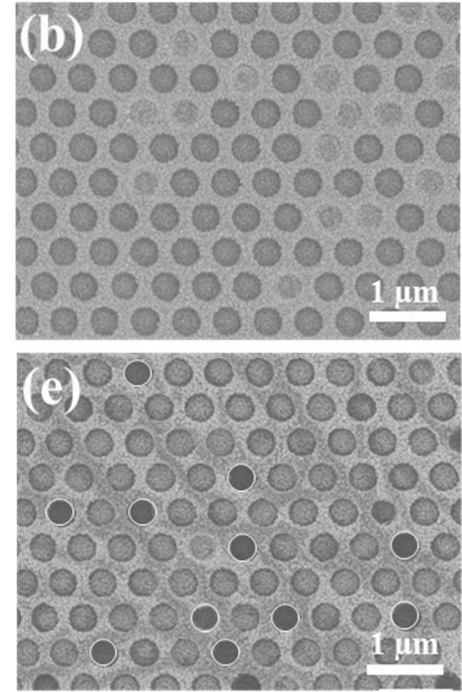
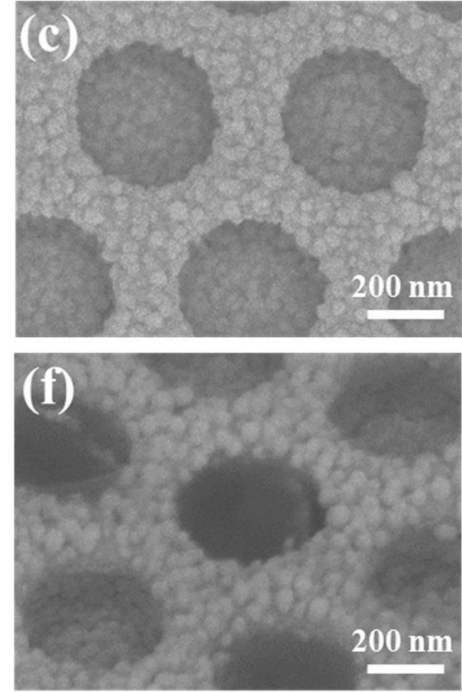

Figure 4. Molecular enrichment and selective deposition in nanowells. (a) Photograph of a water droplet on the quasi-3D plasmonic nanowell substrate. The inset shows a profile of the droplet with a contact angle of $134^{\circ}$. (b-f) SEM images of the plasmonic nanowell array taken after complete evaporation of water droplet containing $\mathrm{MB}$, where the images were taken at the central area of the substrate $(\mathbf{b}, \mathbf{c})$, the boundary between the ring-shaped stain and outside $(\mathbf{d})$, and the ring $(\mathbf{e}, \mathbf{f})$. The white circles in (e) indicate dark nanowells that contain a relatively thick MB overcoat. The SEM image of (f) is a $45^{\circ}$-tilted view of the nanowell containing the thick MB overcoat.

As proof-of-concept, we used the optimized plasmonic chips to detect lactic acid. Lactic acid accumulates between cells unless the tissue is supplied with enough oxygen to support aerobic oxidation of glucose. Because the concentration of lactic acid in the blood is related to the rate of muscle cell production and liver metabolism, lactic acid has been widely used as an indicator of muscle tolerance $[27,33]$. A $3 \mu \mathrm{L}$ of lactic acid aqueous solution with six different concentrations, from 0.5 to $0.01 \mathrm{mM}$ were dropped on the surface of the quasi-3D SERS substrate, and then the solvent was evaporated. The Raman spectra were recorded by measuring five random spots of quasi-3D nanowell structures for $0.5 \mathrm{mM}$ concentration, using a $633 \mathrm{~nm}$ portable Raman spectrometer (Figure $5 \mathrm{a}$ ). The characteristic peak of lactic acid at $869 \mathrm{~cm}^{-1}$ peak was used to assess the signal uniformity [27]. The RSD was as small as $6.8 \%$ for $869 \mathrm{~cm}^{-1}$. Once again, we confirmed the high signal uniformity of the 3D plasmonic nanostructures. Figure $5 \mathrm{~b}$ shows quantitative analysis of lactic acid on 3D plasmonic nanostructures. The SERS intensity of Raman band at $869 \mathrm{~cm}^{-1}$ changes proportionally with lactic acid concentration. For $0.01 \mathrm{mM}$, no characteristic peaks are detected and only background signal from dielectric interlayer is detected [5], indicating that the LOD is measured to be $0.05 \mathrm{mM}$, which is 6 times lower than the LOD in the previous study [27]. A calibration plot with four known concentrations ranging from $0.5 \mathrm{mM}$ to $0.05 \mathrm{mM}$ was obtained from with a good correlation coefficient of 0.98 (Figure 5c). 
(a)

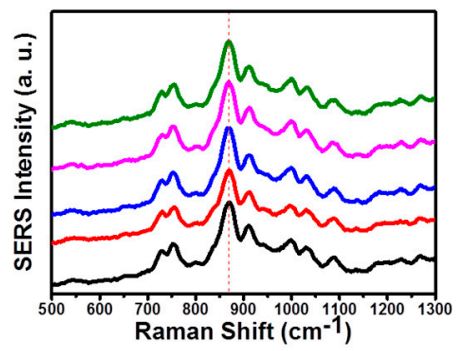

(b)

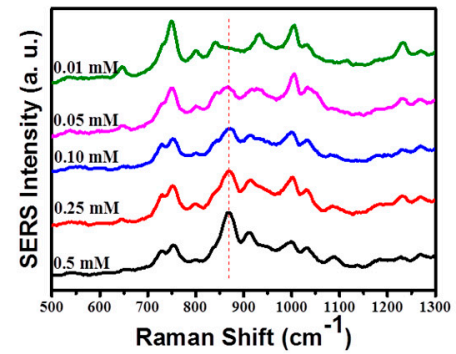

(c)

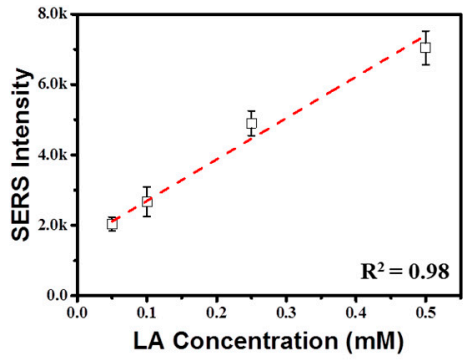

Figure 5. (a) Raman spectra of $0.5 \mathrm{mM}$ lactic acid (LA) measured from the five random spots of quasi-3D nanowell structures. The characteristic peak of lactic acid at $869 \mathrm{~cm}^{-1}$ peak was used to assess the signal uniformity. The RSD was as small as $6.8 \%$ at $869 \mathrm{~cm}^{-1}$. (b) Comparison of the Raman spectra of lactic acid obtained from $0.5 \mathrm{mM}$ to $0.01 \mathrm{mM}$ lactic acid-treated 3D plasmonic nanostructures. (c) Calibration plot with four known concentrations. The SERS intensity at $869 \mathrm{~cm}^{-1}$ was used for the calibration plot.

\section{Conclusions}

In this work, we fabricated quasi-3D plasmonic nanowell structures by combining highly reproducible, scalable, and cost-effective NIL and 3D growth of Au NPs on an ultra-low-energy surface. Au NPs grown on the sidewall and bottom surface of nanowell formed strong hotspots at the nanogaps with the Au-coated surfaces and interstitial voids, amplifying Raman signal for the molecules deposited on the hotspots and thereby providing highly sensitive molecular detection. Moreover, the quasi-3D nanowell array exhibited a very uniform Raman signal, with a relative standard deviation as small as 5.3\% in microscopic mapping analysis, as the quasi-3D plasmonic nanostructures were uniformly created with a negligible structural variation. An analyte can be concentrated and selectively deposited in nanowells by drying an analyte-dissolved droplet, which potentially improves Raman sensitivity. We believe that the combination of high sensitivity and signal uniformity as well as reproducible and low-cost production will provide a new opportunity for translating prominent plasmonic technologies toward commercialization, especially in the area of biosensors.

Supplementary Materials: The following are available online at http://www.mdpi.com/2079-4991/10/5/939/s1, Figure S1: SEM images of the Au nanowells whose surfaces are decorated with Au NPs using deposition thickness of (a) $25 \mathrm{~nm}$, (b) $50 \mathrm{~nm}$, and (c) $75 \mathrm{~nm}$. (d) Histogram of the size distributions of spherical Au NPs decorated on $\mathrm{Au}$ nanowells for the three different deposition conditions, as denoted. The solid lines are fitting curves with the Gaussian function.

Author Contributions: S.-G.P. designed and directed the research; S.K., C.M., and H.S.J. fabricated 3D nanowell arrays and performed the experiments; S.-G.P., D.-H.K., D.-G.C. and S.-H.K. revised and discussed the results; S.-G.P. and S.K. wrote the manuscript and S.-H.K. revised it. All authors have read and agreed to the published version of the manuscript.

Funding: This research was supported by the Fundamental Research Program (PNK 6800) of the Korean Institute of Materials Science (KIMS). This work was supported by the "Ministry of Trade, Industry and Energy" (Grant N0002310). This research was supported by the "Nano Product Upgrading Program using Electron Beam" through the Gyeongsangnam-do and Gimhae.

Conflicts of Interest: The authors declare no conflict of interest.

\section{References}

1. Mun, C.; Linh, V.T.N.; Kwon, J.-D.; Jung, H.S.; Kim, D.-H.; Park, S.-G. Highly Sensitive and Selective Nanogap-Enhanced SERS Sensing Platform. Nanomaterials 2019, 9, 619. [CrossRef]

2. Park, S.-G.; Mun, C.; Xiao, X.; Braun, A.; Kim, S.; Giannini, V.; Maier, S.A.; Kim, D.-H. Surface Energy-Controlled SERS Substrates for Molecular Concentration at Plasmonic Nanogaps. Adv. Funct. Mater. 2017, 27, 1703376. [CrossRef] 
3. Lee, M.; Jeon, T.Y.; Mun, C.; Kwon, J.-D.; Yun, J.; Kim, S.-H.; Kim, D.-H.; Chang, S.-C.; Park, S.-G. 3D multilayered plasmonic nanostructures with high areal density for SERS. RSC Adv. 2017, 7, 17898-17905. [CrossRef]

4. Wang, X.; Park, S.-G.; Ko, J.; Xiao, X.; Giannini, V.; Maier, S.A.; Kim, D.-H.; Choo, J. Sensitive and Reproducible Immunoassay of Multiple Mycotoxins Using Surface-Enhanced Raman Scattering Mapping on 3D Plasmonic Nanopillar Arrays. Small 2018, 14, e1801623. [CrossRef]

5. Park, S.-G.; Xiao, X.; Min, J.; Mun, C.; Jung, H.S.; Giannini, V.; Weissleder, R.; Maier, S.A.; Im, H.; Kim, D. Self-Assembly of Nanoparticle-Spiked Pillar Arrays for Plasmonic Biosensing. Adv. Funct. Mater. 2019, 29, 29. [CrossRef]

6. Jeon, T.Y.; Park, S.-G.; Kim, S.-H.; Kim, D.J.; Kim, D.-H. Nanostructured plasmonic substrates for use as SERS sensors. Nano Converg. 2016, 3, 2957. [CrossRef] [PubMed]

7. Ko, J.; Park, S.-G.; Lee, S.; Wang, X.; Mun, C.; Kim, S.; Kim, D.-H.; Choo, J. Culture-Free Detection of Bacterial Pathogens on Plasmonic Nanopillar Arrays Using Rapid Raman Mapping. ACS Appl. Mater. Interfaces 2018, 10, 6831-6840. [CrossRef] [PubMed]

8. Linh, V.T.N.; Xiao, X.; Jung, H.S.; Giannini, V.; Maier, S.A.; Kim, D.-H.; Lee, Y.-I.; Park, S.-G. Compact Integration of $\mathrm{TiO}_{2}$ Nanoparticles into the Cross-Points of 3D Vertically Stacked Ag Nanowires for Plasmon-Enhanced Photocatalysis. Nanomaterials 2019, 9, 468. [CrossRef] [PubMed]

9. Wang, Z.; Zong, S.; Wu, L.; Zhu, D.; Cui, Y. SERS-Activated Platforms for Immunoassay: Probes, Encoding Methods, and Applications. Chem. Rev. 2017, 117, 7910-7963. [CrossRef] [PubMed]

10. Ionescu, R.E.; Aybeke, E.N.; Bourillot, E.; Lacroute, Y.; Lesniewska, E.; Adam, P.; Bijeon, J.-L. Fabrication of Annealed Gold Nanostructures on Pre-Treated Glow-Discharge Cleaned Glasses and Their Used for Localized Surface Plasmon Resonance (LSPR) and Surface Enhanced Raman Spectroscopy (SERS) Detection of Adsorbed (Bio)molecules. Sensors 2017, 17, 236. [CrossRef]

11. Aybeke, E.N.; Belliot, G.; Lemaire-Ewing, S.; Estienney, M.; Lacroute, Y.; Pothier, P.; Bourillot, E.; Lesniewska, E. HS-AFM and SERS Analysis of Murine Norovirus Infection: Involvement of the Lipid Rafts. Small 2016, 13, 1600918. [CrossRef] [PubMed]

12. Punj, D.; Mivelle, M.; Moparthi, S.B.; Van Zanten, T.; Rigneault, H.; Van Hulst, N.F.; Garcia-Parajo, M.F.; Wenger, J. A plasmonic 'antenna-in-box' platform for enhanced single-molecule analysis at micromolar concentrations. Nat. Nanotechnol. 2013, 8, 512-516. [CrossRef] [PubMed]

13. Li, J.-F.; Li, C.-Y.; Aroca, R.F. Plasmon-enhanced fluorescence spectroscopy. Chem. Soc. Rev. 2017, 46, 3962-3979. [CrossRef] [PubMed]

14. Jeon, H.C.; Heo, C.-J.; Lee, S.Y.; Park, S.-G.; Yang, S.-M. Optically tunable arrayed structures for highly sensitive plasmonic detection via simplified holographic lithography. J. Mater. Chem. 2012, 22, 4603. [CrossRef]

15. Jeon, H.C.; Park, S.-G.; Cho, S.; Yang, S.-M. Dual length-scale nanotip arrays with controllable morphological features for highly sensitive SERS applications. J. Mater. Chem. 2012, 22, 23650. [CrossRef]

16. Park, S.-G.; Yang, S.-M. Multicolor patterning using holographic woodpile photonic crystals at visible wavelengths. Nanoscale 2013, 5, 4110. [CrossRef]

17. Park, S.-G.; Jeon, T.Y.; Jeon, H.C.; Yang, S.-M.; Kwon, J.-D.; Mun, C.-W.; Cho, B.; Kim, C.S.; Kim, D.-H. Fabrication of 3D ZnO hollow shell structures by prism holographic lithography and atomic layer deposition. J. Mater. Chem. C 2014, 2, 1957-1961. [CrossRef]

18. Park, S.-G.; Lee, S.-K.; Moon, J.H.; Yang, S.-M. Holographic fabrication of three-dimensional nanostructures for microfluidic passive mixing. Lab Chip 2009, 9, 3144. [CrossRef]

19. Park, S.-G.; Moon, J.H.; Lee, S.-K.; Shim, J.; Yang, S.-M. Bioinspired Holographically Featured Superhydrophobic and Supersticky Nanostructured Materials. Langmuir 2010, 26, 1468-1472. [CrossRef]

20. Park, S.-G.; Miyake, M.; Yang, S.-M.; Braun, P.V. $\mathrm{Cu}_{2} \mathrm{O}$ Inverse Woodpile Photonic Crystals by Prism Holographic Lithography and Electrodeposition. Adv. Mater. 2011, 23, 2749-2752. [CrossRef]

21. Kim, A.; Barcelo, S.J.; Li, Z. SERS-based pesticide detection by using nanofinger sensors. Nanotechnology 2014, 26, 15502. [CrossRef] [PubMed]

22. Ou, F.S.; Hu, M.; Naumov, I.; Kim, A.; Wu, W.; Bratkovsky, A.M.; Li, X.; Williams, R.S.; Li, Z. Hot-Spot Engineering in Polygonal Nanofinger Assemblies for Surface Enhanced Raman Spectroscopy. Nano Lett. 2011, 11, 2538-2542. [CrossRef] [PubMed] 
23. Kim, A.; Ou, F.S.; Ohlberg, D.A.A.; Hu, M.; Williams, R.S.; Li, Z. Study of Molecular Trapping Inside Gold Nanofinger Arrays on Surface-Enhanced Raman Substrates. J. Am. Chem. Soc. 2011, 133, 8234-8239. [CrossRef] [PubMed]

24. Kim, A.; Barcelo, S.J.; Williams, R.S.; Li, Z. Melamine Sensing in Milk Products by Using Surface Enhanced Raman Scattering. Anal. Chem. 2012, 84, 9303-9309. [CrossRef]

25. Chen, J.; Li, Y.; Huang, K.; Wang, P.; He, L.; Carter, K.; Nugen, S.R. Nanoimprinted Patterned Pillar Substrates for Surface-Enhanced Raman Scattering Applications. ACS Appl. Mater. Interfaces 2015, 7, 22106-22113. [CrossRef]

26. Chou, S.Y.; Krauss, P.R.; Renstrom, P.J. Imprint of sub-25 nm vias and trenches in polymers. Appl. Phys. Lett. 1995, 67, 3114-3116. [CrossRef]

27. Hsu, P.-H.; Chiang, H.K. Surface-enhanced Raman spectroscopy for quantitative measurement of lactic acid at physiological concentration in human serum. J. Raman Spectrosc. 2010, 41, 1610-1614. [CrossRef]

28. Dalvi, V.; Rossky, P.J. Molecular origins of fluorocarbon hydrophobicity. Proc. Natl. Acad. Sci. USA 2010, 107, 13603-13607. [CrossRef]

29. Love, J.C.; Estroff, L.A.; Kriebel, J.K.; Nuzzo, R.G.; Whitesides, G.M. Self-Assembled Monolayers of Thiolates on Metals as a Form of Nanotechnology. Chem. Rev. 2005, 105, 1103-1170. [CrossRef]

30. Campbell, C.T. Ultrathin metal films and particles on oxide surfaces: Structural, electronic and chemisorptive properties. Surf. Sci. Rep. 1997, 27, 1-111. [CrossRef]

31. Lee, S.-K.; Park, S.-G.; Moon, J.H.; Yang, S.-M. Holographic fabrication of photonic nanostructures for optofluidic integration. Lab Chip 2008, 8, 388. [CrossRef] [PubMed]

32. Lee, M.; Mun, C.; Kim, D.-H.; Chang, S.-C.; Park, S.-G. Analyte-concentrating 3D hybrid plasmonic nanostructures for use in highly sensitive chemical sensors. RSC Adv. 2016, 6, 92120-92126. [CrossRef]

33. Fletcher, W.M.; Hopkins, F.G. Lactic acid in amphibian muscle1. J. Physiol. 1907, 35, 247-309. [CrossRef] [PubMed]

(C) 2020 by the authors. Licensee MDPI, Basel, Switzerland. This article is an open access article distributed under the terms and conditions of the Creative Commons Attribution (CC BY) license (http://creativecommons.org/licenses/by/4.0/). 